\title{
Reduction of Sympathetic Inotropic Response After Ischemia in Dogs Contributor to Stunned Myocardium
}

Allen A. Ciuffo, Pamela Ouyang, Lewis C. Becker, Lisa Levin, and Myron L. Weisfeldt

Division of Cardiology, Department of Medicine, The Johns Hopkins Hospital, and the Division of Cardiology, Department of Medicine, Francis Scott Key Medical Center, Baltimore, Maryland 21205

\section{Abstract}

Eight open chest dogs underwent 25 min of coronary occlusion to determine whether brief myocardial ischemia disrupts the normal myocardial inotropic response to sympathetic nervous stimulation. If so, this could represent a mechanism contributing to postischemic myocardial dysfunction.

Myocardial segment shortening was measured using ultrasonic dimension crystals before and after coronary artery occlusion and reperfusion. Left ansa subclavia stimulation and systemic norepinephrine (NE) infusion were used to test the myocardial inotropic response to neural stimulation and direct exposure to the sympathetic mediator, respectively.

Before coronary artery occlusion, base-line preischemic segment shortening $(12.5 \pm 1.6 \%)$ (SEM) increased during both sympathetic stimulation (20.2 $\pm 1.4 \%)$ and $\mathrm{NE}$ infusion (19.7 $\pm 1.1 \%)$. The control segment responded similarly. After ischemia and reperfusion there was no significant change in heart rate, aortic or left ventricular pressures, nor changes in control segment shortening. In contrast, shortening in the postischemic segment was markedly reduced compared to baseline $(4.1 \pm 2.4 \%)$, and no longer responded to sympathetic stimulation $(2.4 \pm 2.8 \%)$, while responsiveness to systemic NE was maintained $(12.9 \pm 2.0 \%), P<0.001$, which suggested injury to the sympathetic-neural axis during the period of ischemia. This reduced response to neural stimulation was persistent for up to $2 \mathrm{~h}$ after reperfusion.

Left atrial or intracoronary infusion of bretylium tosylate, which releases norepinephrine from nerve terminals, resulted in an immediate inotropic response in the postischemic segment, which indicated that total depletion of $\mathrm{NE}$ from nerve terminals during the ischemic period had not occurred.

Disruption of sympathetic neural responsiveness is likely a component of the mechanism of postischemic myocardial dysfunction whenever there is appreciable sympathetic drive to the heart.

\section{Introduction}

The introduction of effective, new techniques for restoring myocardial blood flow during acute myocardial ischemia has

This paper was presented in part at the 1983 American Heart Association Meetings in Anaheim, CA.

Address reprint requests to Dr. Ciuffo, Division of Cardiology, Johns Hopkins Hospital.

Received for publication 14 August 1985 and in revised form 7 December 1985.

J. Clin. Invest.

(C) The American Society for Clinical Investigation, Inc.

0021-9738/85/05/1504/06\$\$1.00

Volume 75, May 1985, 1504-1509 led to a great deal of interest in the phenomenon of myocardial dysfunction in postischemic but viable myocardium. Functional impairment in such noninfarcted tissue has been associated with biochemical, ultrastructural, and other functional and morphologic abnormalities of myocardium that persist despite the reestablishment of coronary blood flow (1-3). In addition to intrinsic myocardial factors, extramyocardial factors may also contribute to postischemic dysfunction. Ischemic damage to local cardiac sympathetic efferents with subsequent alterations in regional myocardial sympathetic tone could be one such factor.

Little is known about the sensitivity of the cardiac sympathetic nerves to ischemia. Sympathetic neurotransmission through an area of transmural infarction has been shown to be interrupted such that myocardium apical to the infarction becomes unresponsive to sympathetic stimulation (4). During regional ischemia the response to neural sympathetic stimulation is impaired, while the response to systemic infusion of the sympathetic mediator, norepinephrine, is maintained (5). Recovery of sympathetic response after ischemia has not been studied. In order to determine whether temporary ischemia followed by reperfusion impairs regional adrenergic neurotransmission, we measured myocardial segmental shortening in response to sympathetic nerve stimulation and norepinephrine $(\mathrm{NE})^{1}$ infusion before and after $25 \mathrm{~min}$ of regional ischemia. By comparing the contractile response to exogenously administered NE and endogenously released neurotransmitter, we could assess the integrity of sympathetic nerve function.

\section{Methods}

The myocardial contractile response to stimulation of the cardiac sympathetic nerves and systemic NE infusion was studied before and after 25 min of regional ischemia in eight open chest dogs. In addition, regional myocardial blood flow using microspheres was measured before and after ischemia, during sympathetic stimulation and systemic $\mathrm{NE}$ infusion.

Eight open chest mongrel dogs of either sex weighing 15-20 kg were anesthetized with intravenous thiamylal $(10-20 \mathrm{mg} / \mathrm{kg})$ and intramuscular chloralose and urethane $(80$ and $800 \mathrm{mg} / \mathrm{kg}$, respectively). After endotracheal intubation, mechanical ventilation was controlled with a Harvard respirator pump. After a left thoracotomy, the heart was suspended in a pericardial cradle. A high fidelity micromanometer tipped catheter (Millar Corp.) was introduced into the left ventricle via the left atrium. Fluid-filled catheters were placed into the descending aorta for measurement of systemic blood pressure and the left atrium for injection of radioactive microspheres. Regional wall motion was quantitated by pairs of ultrasonic dimension crystals, placed into the mid-myocardium and oriented to measure fiber shortening perpendicular to the long axis of the left ventricle. At constant temperature the drift of these ultrasonic dimension gauges is $<0.01 \mathrm{~mm}$ in $6 \mathrm{~h}(6)$. Segmental

1. Abbreviations used in this paper: $\mathrm{dP} / \mathrm{dt}$, left ventricular stroke work; NE, norepinephrine. 
wall motion was expressed as percentage shortening: (end diastolic length-end systolic length)/([end diastolic length] $\times 100 \%)$. End diastolic length was measured just before isovolumic contraction, which was determined by the left ventricular pressure wave form. End systolic length was measured $20 \mathrm{~ms}$ before the peak negative left ventricular stroke work $(\mathrm{dP} / \mathrm{dt})$.

The left ansa subclavia nerve was carefully isolated by blunt and sharp dissection. Bipolar stimulating electrodes were placed around the ansa subclavia and stimulation established by delivery of a square wave pulse of 6-12 V, 10-20 Hz, and 10-20 ms from a Grass S5 stimulator. Evidence of stimulation was readily apparent by a rapid increase in blood pressure, heart rate, and segmental shortening, and steady state was achieved in $<10-15 \mathrm{~s}$.

Myocardial blood flow was measured using radioactive microspheres. For each measurement, approximately two million $15 \pm 3$ micron microspheres labeled with ${ }^{95} \mathrm{Nb},{ }^{46} \mathrm{Sc},{ }^{141} \mathrm{Ce},{ }^{103} \mathrm{Ru}$, or ${ }^{113} \mathrm{Sn}$ were injected into the left atrium. Beginning $15 \mathrm{~s}$ before each injection and continuing for $2 \mathrm{~min}$, a reference blood sample was obtained from the femoral artery by constant withdrawal. After killing, hearts were fixed in $10 \%$ formalin and cut into $0.5-1-\mathrm{cm}$ thick rings perpendicular to the long axis of the heart. Samples weighing 1-2 $\mathrm{g}$ were taken from the regions circumscribed by the postischemic and control segments. Samples were divided into endocardial and epicardial halves, weighed and counted in a gamma scintillation counter. Raw counts were corrected for background activity and energy crossover and compared with the reference sample to obtain blood flow as previously described (6).

Experimental protocol. After lidocaine, $2-3 \mathrm{mg} / \mathrm{kg}$ i.v., the left anterior descending $(n=7)$ or circumflex coronary artery $(n=1)$ was dissected distally, beyond the last significant diagonal or marginal branch, well below the sonomicrometer crystals, and ligated. A level of supramaximal sympathetic stimulation was established by stepwise increments in voltage until no further increase in segmental shortening was noted. Stimulation parameters were then fixed and not changed for the duration of the experiment. Similarly, the heart was paced from the left atrium at the heart rate obtained during supramaximal sympathetic stimulation $(150-175 / \mathrm{min})$ and the pacing rate was not altered during any subsequent measurement period.

After measurements of base-line values of aortic and left ventricular pressure, heart rate, and segmental shortening, sympathetic stimulation was carried out for $30 \mathrm{~s}$ and measurements repeated immediately after discontinuation of nerve stimulation. Sympathetic stimulation resulted in an abrupt increase in blood pressure, $\mathrm{dP} / \mathrm{dt}$, and segmental shortening in both segments. After return to baseline, which required $<2 \mathrm{~min}$, intravenous NE infusion was begun and titrated to a dose sufficient to result in a degree of shortening in the preischemic segment similar to that seen in this segment during sympathetic stimulation (NE dose range $0.5-1.0 \mu \mathrm{g} / \mathrm{kg}$ per $\mathrm{min}$ ). This dose of NE was noted and used for all further measurements of NE response throughout the experiment. Norepinephrine was then discontinued and, after a return of measured parameters to baseline, 3,000 $\mathrm{U}$ of heparin were administered intravenously. A $2.5 \mathrm{~F}$ balloon tipped catheter was then passed retrograde from the previously dissected and ligated distal coronary artery and the balloon inflated to occlude coronary flow, such that one set of crystals was included in the resulting hypoperfused zone (Fig. 1).

Intraluminal balloon occlusion via the retrograde approach was chosen as the technique for coronary artery occlusion after pilot studies demonstrated complete loss of enhancement of segmental shortening by sympathetic neural stimulation in crystal pairs located in the anterior wall of the left ventricle after blunt dissection of a 5-mm segment of the proximal left anterior descending coronary artery even without coronary occlusion. This was attributed to mechanical disruption of the cardiac sympathetic nerves in their course along the proximal coronary vessel, a problem that could be avoided by using the intraluminal occlusion approach $(7,8)$. The potential for surgical disruption of pericoronary nerves may be unique to the left anterior descending coronary artery, as others have not found this to occur after dissection of the circumflex coronary artery (9).

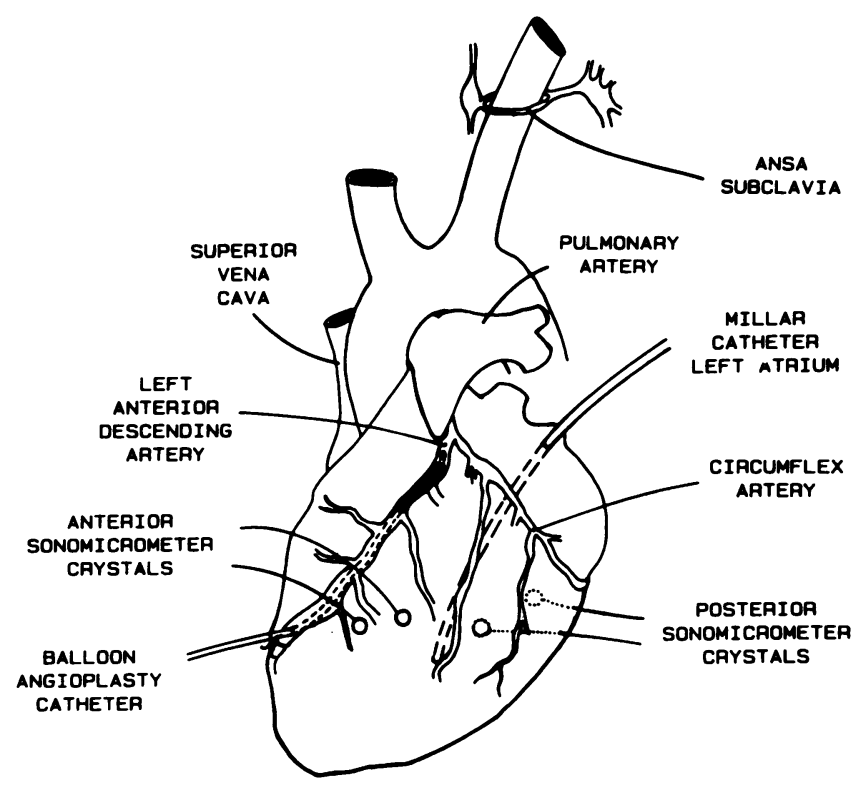

Figure 1. Diagram of experimental preparation. Anterior and posterior ultrasonic dimension crystals are placed in the midmyocardium. To avoid potential damage to the pericoronary cardiac sympathetic fibers, the left anterior descending coronary artery is occluded with an angioplasty balloon introduced distal to the last significant diagonal branch.

After $25 \mathrm{~min}$ of ischemia, the balloon was deflated and the catheter removed. $5 \mathrm{~min}$ later, base-line values were again measured and the sympathetic stimulation and NE protocols repeated. Myocardial blood flow was measured at baseline before ischemia, $15 \mathrm{~min}$ after coronary artery occlusion, $10 \mathrm{~min}$ after reperfusion, and during postischemic sympathetic stimulation and NE infusion.

After the above protocol, seven other dogs underwent infusion of bretylium tosylate. A bolus of bretylium was injected into the left atrium (20 mg, $n=5$ ), or directly into the coronary artery supplying the postischemic segment $(0.1-0.5 \mathrm{mg}, n=4)$, and changes in systemic and left ventricular blood pressure and segmental shortening were measured continuously for $2 \mathrm{~min}$. After the last measurement, dogs were killed and the hearts fixed in formalin for later sectioning and microsphere blood flow determination.

Statistics. Data were analyzed using repeated measures analysis of variance with multiple comparisons where indicated (Neuman-Keels multiple comparisons test). Results are presented as mean \pm SEM.

\section{Results}

Hemodynamics. There was no significant change in left ventricular end diastolic pressure before or after ischemia during any of the measurement periods (Table I). Peak left ventricular systolic pressure at baseline was unchanged before and after $25 \mathrm{~min}$ of ischemia. Peak aortic pressure rose to a similar degree before and after the ischemic period during both sympathetic stimulation and NE infusion. Peak systolic pressure during sympathetic stimulation, however, was less than during NE infusion both before and after ischemia (Table I). Heart rate was held constant throughout by atrial pacing.

Segment length changes. Fig. 2 shows recordings of segment length from a single representative experiment. Vertical ticks drawn on the tracing indicate end diastole and end systole determined from the left ventricular pressure tracing and the peak negative $\mathrm{dP} / \mathrm{dt}$. Base-line shortening in the control segment 


\begin{tabular}{|c|c|c|c|c|c|}
\hline & & Baseline & Ansa subclavia & Norepinephrine & \\
\hline & & & & & $P$ \\
\hline pressure $(\mathrm{mmHg})$ & Postischemic & $7.4 \pm 1.2$ & $5.7 \pm 1.1$ & $6.9 \pm 0.9$ & NS \\
\hline Peak left ventricular & Preischemic & $112 \pm 6$ & $143 \pm 6$ & $170 \pm 10$ & $<0.005$ \\
\hline pressure $(\mathrm{mmHg})$ & Postischemic & $108 \pm 7$ & $135 \pm 8$ & $165 \pm 9$ & $<0.005$ \\
\hline
\end{tabular}

was essentially unchanged before and after ischemia. During sympathetic stimulation and NE infusion, shortening increased to a similar extent before and after ischemia. The response in the postischemic segment, however, was considerably different. After coronary artery occlusion and reflow, base-line shortening of the postischemic segment was markedly reduced. The response to norepinephrine infusion, however, was maintained. In contrast to the persistence of response to norepinephrine, the response to sympathetic nerve stimulation was abolished. In this example, the postischemic segment demonstrated systolic lengthening rather than shortening, as well as a midsystolic bulge during sympathetic stimulation, with shortening occurring only late, well after the end of systole.

Before coronary artery occlusion, for all eight experiments, mean shortening at baseline $(12.5 \pm 1.6 \%)$ increased to a similar extent during sympathetic stimulation $(20.2 \pm 1.4 \%)$ and $\mathrm{NE}$ infusion $(19.7 \pm 1.1 \%)$ (Fig. 3). In the control segment, baseline shortening $(6.9 \pm 1.4 \%)$ also increased during sympathetic nerve stimulation $(14.8 \pm 2.0 \%)$, and during $\mathrm{NE}$ infusion $(10.1 \pm 1.6 \%)$ (Fig. 4$)$

After $25 \mathrm{~min}$ of proximal coronary artery, occlusion and reflow measurements were repeated. The control segment continued to shorten normally at baseline $(9.7 \pm 1.6 \%)$, and there was no change in the response to sympathetic stimulation $(16.4 \pm 1.8 \%)$ and $\mathrm{NE}$ infusion (13.7 $\pm 1.5 \%)$ (Fig. 4). In the postischemic segment (Fig. 3), shortening at baseline was dramatically reduced $(4.1 \pm 2.4 \%)$ after ischemia, and this segment no longer responded to sympathetic stimulation $(2.4 \pm 2.8 \%)$. In contrast, response to NE infusion was retained $(12.9 \pm 2.0 \%)$. Although shortening was reduced in the postischemic segment during baseline, sympathetic stimulation, and $\mathrm{NE}$ infusion periods, the attenuation in response of this segment to sympathetic neural stimulation was significantly greater than that to exogenous norepinephrine, $P<0.001$. In five of these animals, as well as in three additional experiments, observations were carried out for up to $120 \mathrm{~min}$ (Fig. 5). These observations confirm that the reduced response to neural stimulation was persistent for at least that duration, despite a retained response to $\mathrm{NE}$

Regional myocardial blood flow. Myocardial blood flow was determined in the regions of the ischemic and control ultrasonic crystal pairs using radioactive microspheres. Regional myocardial blood flow was not changed in the control region during ischemia, was increased somewhat over baseline 10

PREISCHEMIC

POST ISCHEMIC

BASELINE

ANSA SUBCLAVIA

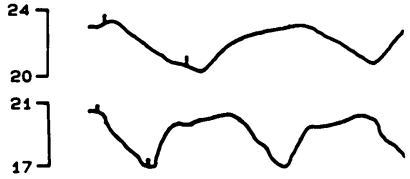

CONTROL SEGMENT
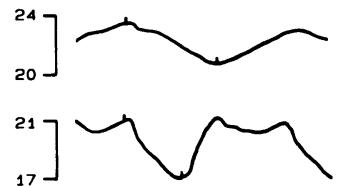

NOREPINEPHAINE
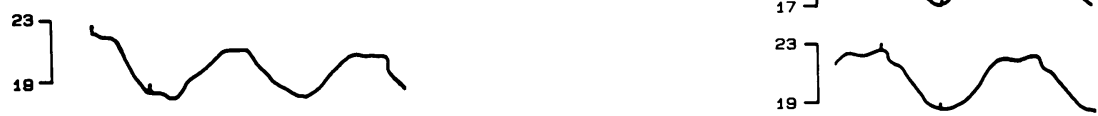

BASELINE

ANSA SUBCLAVIA
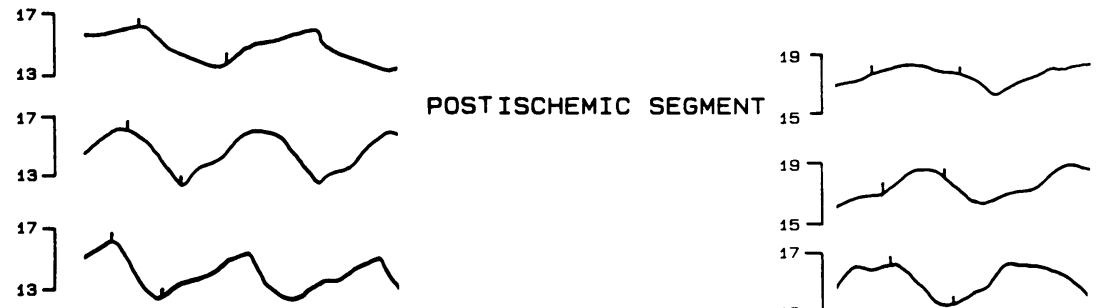

NOREPINEPHAINE

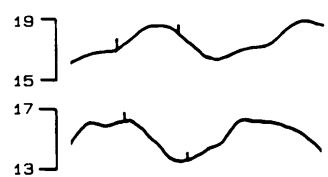

Figure 2. Data from representative experiment. Vertical ticks mark end systole and end diastole. After ischemia and reperfusion, sympathetic stimulation no longer results in augmented shortening in the postischemic segment, while the response to nerve stimulation is

normal in the control segment. Despite the lack of response to nerve stimulation after ischemia, responsiveness to NE infusion is maintained. 


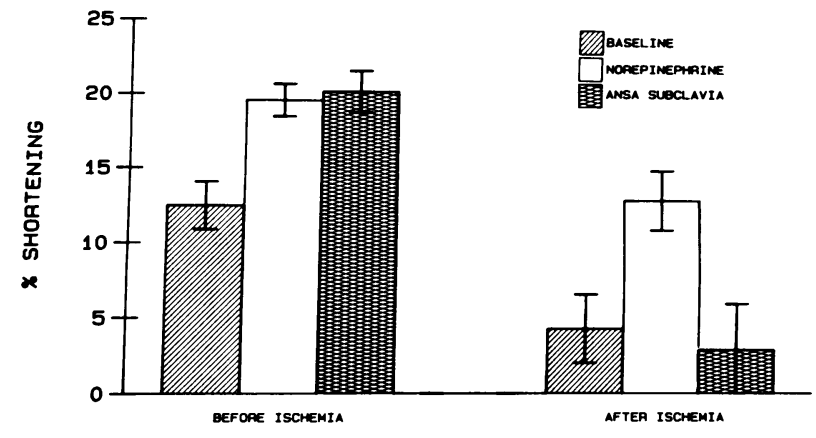

Figure 3. Segmental shortening in postischemic segments. Shortening was augmented equally during NE infusion and ansa subclavia stimulation before the ischemic period. After $25 \mathrm{~min}$ of ischemia and reperfusion, base-line function was reduced. While response to norepinephrine was maintained, the segment was no longer responsive to ansa subclavia stimulation $(n=8)$.

min after reflow, and increased further during sympathetic stimulation and norepinephrine infusion, $P<0.05$ (Fig. 6). Myocardial blood flow to the postischemic segment (Fig. 7) was reduced during the ischemic period, and did not increase significantly during sympathetic stimulation nor during NE infusion. In three hearts, myocardial necrosis was assessed after 3-4 h of reperfusion by tetrazolium staining (10). Infarction was limited to the distal apex, well below the anterior segment crystal pair, and involved $<5 \%$ of left ventricular mass.

Bretylium. Bretylium tosylate releases NE from nerve terminals in the myocardium (11). The lack of an inotropic response to bretylium, with a preserved response to exogenous $\mathrm{NE}$, would suggest that regional depletion of NE had occurred. Infusion of bretylium tosylate into the region of the postischemic segment either via the left atrium $(n=5)$ or directly into the coronary artery $(n=4)$ supplying that segment resulted in an abrupt increase in segmental shortening in all cases. In this group, before ischemia, base-line segmental shortening $(15.8 \pm 2.6 \%)$ was enhanced after sympathetic stimulation $(21.0 \pm 3.4 \%)$ and NE infusion $(23.2 \pm 3.4 \%)$. After $25 \mathrm{~min}$ of intraluminal coronary artery occlusion and reperfusion, resting function was reduced $(8.2 \pm 1.2 \%)$ and no longer responded to sympathetic stimulation $(8.0 \pm 1.3 \%)$, but continued to respond to NE infusion $(16.4 \pm 1.4 \%)$. Left atrial infusion of bretylium

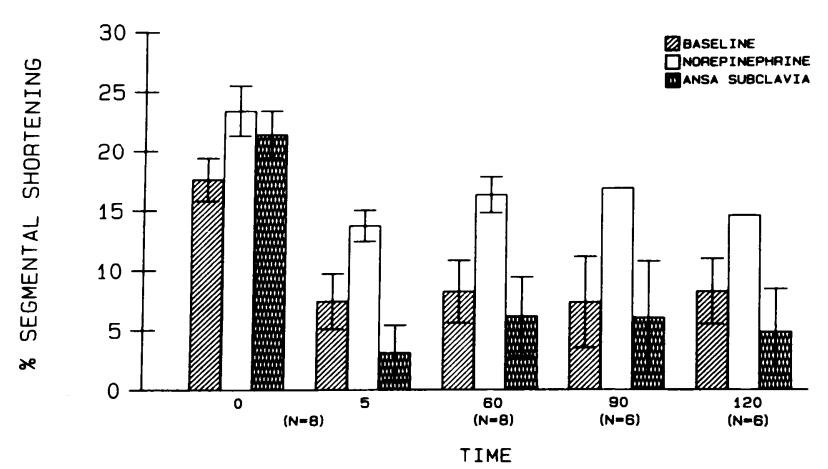

Figure 5. Segmental shortening in the postischemic segment 5, 60, and $120 \mathrm{~min}$ after reperfusion. Baseline and ansa subclavia stimulation function were measured in six animals up to $120 \mathrm{~min}$. NE response was measured in only two animals at 90 and $120 \mathrm{~min}$. The reduction in response to neural stimulation is persistent despite maintained response to $\mathrm{NE}$ infusion.

enhanced segmental shortening to a similar degree as exogenous $\mathrm{NE}$ infusion in the postischemic segment $(20.7 \pm 2.5 \%)$. Left atrial bretylium also increased control segment shortening from $11.8 \pm 1.3$ to $17.8 \pm 0.5 \%$. To control for the possibility of a systemic effect of bretylium, five animals received intracoronary bretylium into the coronary artery serving the postischemic segment. In this group, shortening in the postischemic segment increased from $6.2 \pm 7.8$ to $18.2 \pm 3.9 \%$ after intracoronary bretylium, while there was no response in the corresponding control segment.

\section{Discussion}

Temporary myocardial ischemia results in prolonged myocardial dysfunction even when there is no evidence of myocardial necrosis. In the present study, the ischemic period was limited to $25 \mathrm{~min}$, a period which has been associated with minimal or no necrosis of canine myocardium (12). Despite the brief period of ischemia there was a dramatic reduction in contractile function and loss of responsiveness of the postischemic myocardium to neural sympathetic stimulation, while responsiveness to exogenous NE was preserved.

Prolonged myocardial dysfunction has been demonstrated after $20 \mathrm{~min}$ of ischemia (13), with lack of recovery for up to $45 \mathrm{~min}$. In conscious dogs, only $15 \mathrm{~min}$ of ischemia followed

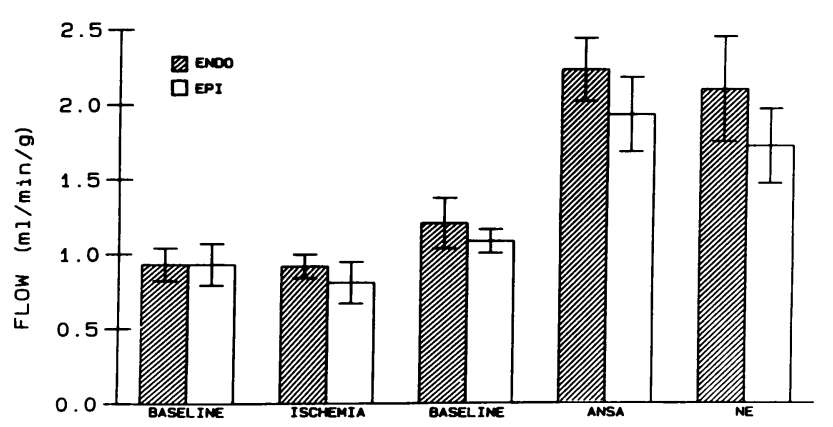

Figure 6. Control segment regional myocardial blood flow. Regional blood flow was somewhat increased in this segment after reperfusion in the distant segment and during NE infusion and ansa subclavia stimulation $(n=8)$.
Figure 4. Segmental shortening in the control segment. Segmental shortening was augmented in the control segment both during NE infusion and during stimulation of the ansa subclavia $(n=8)$. 


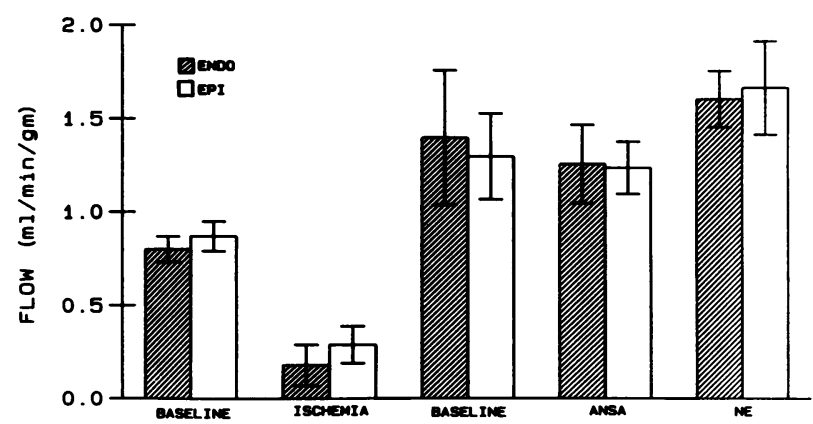

Figure 7. Postischemic segment regional myocardial blood flow. Regional blood flow was markedly reduced from control during the ischemic period. There was no reduction in flow after reperfusion, during NE infusion, or during ansa subclavia (ansa) stimulation $(n=8)$.

by reperfusion results in $3 \mathrm{~h}$ of severe regional myocardial dysfunction, with return to normal function after $24 \mathrm{~h}$ (14). The pathogenesis of this prolonged contractile dysfunction after brief ischemia has been related to biochemical, ultrastructural, and other functional and morphologic abnormalities of postischemic myocardium (15). The results of the present study show that with the severity and duration of ischemia employed, these factors do not influence functional performance of myocardium sufficiently to markedly reduce the inotropic state of the postischemic myocardium during inotropic stimulation by exogenous norepinephrine. Thus, these factors may not contribute to dysfunction of postischemic myocardium without longer, more severe, or repeated periods of ischemia.

Little is known about the sensitivity of the cardiac sympathetic nerves to ischemia. In skeletal muscle, morphologic evidence of ischemic damage can be seen in sympathetic nerves after $2 \mathrm{~h}$ of ischemia, while ischemic changes in myocytes are not evident until at least $3 \mathrm{~h}$ (16). In rabbit heart, partial depletion of myocardial catecholamine stores becomes evident after only $30 \mathrm{~min}$ of ischemia, and progresses to near total depletion after 2.5-5 h (17).

The functional effect of ischemia on the myocardial response to stimulation of the cardiac sympathetics may become evident much earlier than these morphologic changes. In canine myocardium, shortly after the onset of acute ischemia, the contractile response to sympathetic neural stimulation is ablated, while responsiveness to exogenous norepinephrine is maintained (5). Dysfunction of regional myocardial sympathetic nerves during acute ischemia may not, however, depend on intrinsic functional damage of the cardiac sympathetics. It could also result from blockade of neurotransmitter release by adenosine (18), hydrogen ion (19), or potassium (20), all locally increased in ischemic myocardium. In noninfarcted myocardium, denervation has been demonstrated in areas lying apical to a transmural infarction, presumably secondary to damage of efferent sympathetic nerves passing through the infarct zone from base to apex (4).

Ablation of cardiac sympathetic nerves via surgical (21) or chemical techniques (22) results in a reduction in resting myocardial contractile function and a loss of response to adrenergic neural stimulation. In the present study, the myocardial response to adrenergic neural transmission was compared with the response to infusion of the neurotransmitter $\mathrm{NE}$ before and after $25 \mathrm{~min}$ of ischemia followed by reperfusion.
An attempt to approximate the same degree of inotropic stimulation with both NE infusion and cardiac neural stimulation was made by titrating the NE dose to result in a similar degree of segment shortening during NE infusion as with neural stimulation. Although ventricular afterload was different with the two forms of sympathetic stimulation, this was probably not sufficient to explain the dramatic difference in response to nerve stimulation and NE infusion after ischemia. The similar response seen in the control segment to both of these stimuli before and after $25 \mathrm{~min}$ of ischemia and in the preischemic segment before ischemia supports this contention.

As expected, the postischemic segment after reperfusion had markedly abnormal function at rest. During nerve stimulation, while the blood pressure and control segmental response were augmented as in the preischemic period, the postischemic segment demonstrated no augmentation in function, and in some segments developed a systolic bulge. In contrast to this, during infusion of norepinephrine both the postischemic and control segments responded with increased segmental shortening over baseline. While the postischemic segment response to infused NE did not reach the preischemic level, function did improve remarkably from the depressed postischemic state. We conclude from these data that sympathetic neural responsiveness is impaired for at least several hours after reperfusion in a transiently ischemic zone.

Microsphere measurements of regional flow were made to determine whether alterations in blood flow to the postischemic segment could be responsible for the reduction in function or response of this segment after reperfusion. Sympathetic neural stimulation as well as NE infusion increased regional myocardial blood flow in the control zone, while there was no statistically significant increase in flow to the postischemic zone after either intervention. The differences in segmental shortening, therefore, after ischemia and reperfusion in response to NE infusion and nerve stimulation did not appear due to measurable changes in the amount or distribution of myocardial blood flow.

The possibility that regional sympathectomy was induced in our preparation by local traumatic damage to the pericoronary nerves which carry the sympathetic efferent and afferent axons was addressed by the method of coronary artery occlusion chosen. Pericoronary dissection has been shown to result in functional and morphologic damage to regional cardiac sympathetic fibers traveling in the superficial epicardium adjacent to coronary arteries $(7,8)$. In the present study, however, coronary occlusion was carried out using a balloon-tipped catheter introduced retrograde into the coronary artery, thereby avoiding trauma to the proximal pericoronary sympathetic nerves.

Although the mechanism of this loss of response to sympathetic stimulation is unknown, it is unlikely to be simple depletion of norepinephrine from the nerve terminals in the ischemic zone. Although ischemia is a profound mediator of release of norepinephrine (23), histochemical studies demonstrate only a small amount of depletion of norepinephrine from nerve terminals after up to $30 \mathrm{~min}$ of ischemia (16).

The inotropic effect of small doses of bretylium is related to rapid release of norepinephrine from nerve terminals (11, 24). If norepinephrine were depleted at the nerve terminals in the postischemic myocardium, exogenous bretylium should have resulted in no immediate response in those areas. The dramatic increase in segmental shortening in the postischemic 
segment after infusion of bretylium, however, indicated that norepinephrine was present in nerve terminals.

Postischemic loss of responsiveness to neural sympathetic tone demonstrated in the present study is likely in part responsible for a reduction in myocardial contractile function observed after brief myocardial ischemia when there is significant sympathetic neural drive to the heart. Dysfunction of a regionally denervated, postischemic segment may be further exaggerated by ischemia-mediated reflexes that enhance sympathetic drive $(25,26)$. Any factor or reflex that might increase cardiac sympathetic activity may be expected to augment dysfunction of the postischemic denervated segment because of the increased stress placed on it by the remainder of the normally innervated, sympathetically driven portions of the ventricle.

The results of the present study show that temporary myocardial ischemia results in loss of myocardial responsiveness to neural sympathetic stimulation, while response to systemic catecholamines is maintained.

\section{Acknowledgments}

This work was supported by an Ischemic Heart Disease Specialized Center of Research Grant (17655-7) from the National Heart, Blood, and Lung Institute.

\section{References}

1. DeBoer, L. W. V., J. S. Ingwall, R. A. Kloner, and E. Braunwald. 1980. Prolonged derangements of canine myocardial purine metabolism following a brief coronary artery occlusion not associated with anatomic evidence of necrosis. Proc. Natl. Acad. Sci. USA. 77:5471-5475.

2. Ellis, S. G., C. I. Henschks, T. Sandor, J. Wynne, E. Braunwald, and R. A. Kloner. 1983. Time course of functional and biochemical recovery of myocardium salvaged by reperfusion. J. Am. Coll. Cardiol. 1(Suppl 4): 1047-1055.

3. Bogen, D. K., S. A. Rabinowitz, A. Needleman, T. A. McMahon, and W. H. Abelman. 1980. An analysis of the mechanical disadvantage of myocardial infarction in the canine left ventricle. Circ. Res. 47(Suppl 5):728-741.

4. Barber, M. K., T. M. Mueller, D. P. Henry, S. Y. Felten, and D. P. Zipes. 1983. Transmural myocardial infarction in the dog produces sympathectomy in noninfarcted myocardium. Circulation. 67:787-796.

5. Martins, J. B., R. E. Kerber, M. L. Marcus, D. L. Laughlin, and D. M. Levy. 1980. Inhibition of adrenergic neurotransmission in ischemic regions of canine left ventricle. Cardiovasc. Res. 14:116-124.

6. Rivas, F., B. Y. Cobb, and J. C. Greenfield. 1976. Relationship between blood flow to ischemic regions and the extent of myocardial infarction. Serial measurements of blood flow to ischemic regions in dogs. Circ. Res. 38(Suppl 5):439-447.

7. Ciuffo, A. A., P. O. Ouyang, L. C. Becker, and M. L. Weisfeldt. 1984. Disruption of pericoronary epicardium results in regional sympathetic denervation of myocardium. Clin. Res. 32:155A. (Abstr.)

8. Dolezel, A., M. Gerova, B. Hartmannova, M. Dostal, H.
Janeckova, and J. Vascu. 1984. Cardiac adrenergic innervation after instrumentation of the coronary artery in dog. Am. J. Physiol. 246: H459-H465.

9. Johanssen, U. J., A. L. Mark, and M. L. Marcus. 1982. Responsiveness to cardiac sympathetic nerve stimulation during maximal coronary dilation produced by adenosine. Circ. Res. 50:510-517.

10. Schaper, J., and W. Schaper. 1983. Reperfusion of ischemic myocardium. Ultrastructural and histochemical aspects. J. Am. Coll. Cardiol. 1:1037-1047.

11. Abbs, E. T., and C. J. Pycock. 1973. The effects of bretylium on the subcellular distribution of noradrenaline and on adrenergic nerve function in rat heart. Br. J. Pharmacol. 49:11-22.

12. Reimer, R. A., M. M. Rasmussen, R. A. Kloner, and R. B. Jennings. 1977. Infarct size reduction by propranolol before and after coronary ligation in dogs. Circulation. 56:784-798.

13. Weiner, J. M., C. S. Apstein, J. H. Arthur, F. A. Pirzada, and W. B. Hood. 1976. Persistence of myocardial injury following brief periods of coronary artery occlusion. Cardiovasc. Res. 10:678-686.

14. Heyndrickx, G. R., H. Baig, P. Nellens, I. Leusen, M. Fishbein, and S. F. Vatner. 1978. Depression of regional blood flow and wall thickening after brief coronary artery occlusions. Am. J. Physiol. 234(Suppl 6):H653-H659.

15. Jennings, R. B. 1969. Early phase of myocardial ischemia injury and infarction. Am. J. Cardiol. 24:753.

16. Teravanian, H., and J. Makitie. 1976. The effect of temporary ischemia on the perivascular sympathetic nerves. Exp. Neurol. 53:178188.

17. Holmgren, S., T. Abrahamsson, O. Almgren, and B.-M. Eriksson. 1981. Effect of ischemia on the adrenergic neurones of the rat heart: a fluorescense histochemical and biochemical study. Cardiovasc. Res. 15:680-689.

18. Verhaeghe, R. H., P. M. Vanhoutte, and J. T. Shepherd. 1977. Inhibition of sympathetic neurotransmission in canine blood vessels by adenosine and adenine nucleotides. Circ. Res. 40:208-215.

19. Ouig, M., and S. M. Kirpekar. 1971. Inhibitory effect of low pH on norepinephrine release. J. Pharmacol. Exp. Ther. 176:134-138.

20. Lorenz, R. R., and P. M. Vanhoutte. 1975. Inhibition of adrenergic neurotransmission in isolated veins of the dog by potassium ions. J. Physiol. 246:479-500.

21. Agrawal, G., P. Silk, E. Glofelty, and B. S. Lefkowitz. 1967. Effect of epicardiectomy on myocardial function. Surgery (St. Louis). 61:399-406.

22. Pace, J. B., W. P. Geis, M. P. Kaye, and D. V. Pirola. 1969. Influence of ventricular epicardiectomy on cardiac response to stellate ganglion stimulation. J. Thorac. Cardiovasc. Surg. 58(Suppl 1):39-47.

23. Abrahamsson, T., O. Almgren, and L. Svensson. 1981. Local noradrenaline release in acute myocardial ischemia: influence of catecholamine synthesis inhibition and beta adrenoreceptor blockade on ischemic injury. J. Cardiovasc. Pharmacol. 3:807-818.

24. Silinsky, E. M. 1974. The effect of bretylium and guanethidine on catecholaminergic transmission in an invertebrate. Br. J. Pharmacol. 51:367-371.

25. Mallaini, A., P. J. Schwartz, and A. Zanchetti. 1968. A sympathetic reflex elicited by experimental coronary occlusion. Am. J. Physiol. 17(Suppl 8):703-709.

26. Mallaini, A., F. Lombardi, and M. Pagani. 1981. Functions of afferents in cardiovascular sympathetic nerves. J. Auton. Nerv. Syst. 3: 231-236. 